\title{
Medical cyber-physical systems: A survey
}

\author{
Nilanjan Dey ${ }^{1}$, Amira S. Ashour ${ }^{2}$, Fuqian $\mathrm{Shi}^{3}$, Simon James Fong ${ }^{4}$, João Manuel R.S. Tavares ${ }^{5}$ \\ ${ }^{1}$ Department of Information Technology, Techno India College of Technology, West Bengal, 740000, \\ India (email: neelanjan.dey@gmail.com) \\ ${ }^{2}$ Department of Electronics and Electrical Communications Engineering, Faculty of Engineering, Tanta \\ University, Egypt (email: amirasashour@yahoo.com) \\ ${ }^{3}$ College of Information \& Engineering, Wenzhou Medical University, Wenzhou, 325035, PR China \\ (e-mail: sfq@wmu.edu.cn) \\ ${ }^{4}$ Department of Computer and Information Science Data Analytics and Collaborative Computing \\ Laboratory University of Macau, Taipa, Macau SAR (ccfong@umac.mo) \\ ${ }^{5}$ Instituto de Ciência e Inovação em Engenharia Mecânica e Engenharia Industrial, Departamento de \\ Engenharia Mecânica, Faculdade de Engenharia, Universidade do Porto, Porto, Portugal (email: \\ tavares@fe.up.pt)
}

\begin{abstract}
Medical cyber-physical systems (MCPS) are healthcare critical integration of a network of medical devices. These systems are progressively used in hospitals to achieve a continuous high-quality healthcare. The MCPS design faces numerous challenges, including inoperability, security/privacy, and high assurance in the system software. In the current work, the infrastructure of the cyber-physical systems (CPS) are reviewed and discussed. This article enriched the researches of the networked Medical Device (MD) systems to increase the efficiency and safety of the healthcare. It also can assist the specialists of medical device to overcome crucial issues related to medical devices, and the challenges facing the design of the medical device's network. The concept of the social networking and its security along with the concept of the wireless sensor networks (WSNs) are addressed. Afterward, the CPS systems and platforms have been established, where more focus was directed toward CPS-based healthcare. The big data framework of CPSs is also included.
\end{abstract}

Keywords - Networked medical device systems, wireless sensor networks, medical internet of things, body area networks, security assurance, patient monitoring.

\section{Introduction}

Cyber physical system (CPS) is an integration of computation, including Cyber world (i.e. computers) and the physical processes through computer networks. Embedded computers and networks are also involved to monitor and control the physical processes through feedback loops [1]. Numerous data types are acquired from a real-world using sensors. These data are then transmitted to the cyber world, processed and analyzed. The idea behind the CPS is to integrate the intelligence in everyday objects/services to execute critical tasks. The CPS concept has a significant role for several information technology (IT) -based social service frameworks [2]. It can be applied to 
social services, especially in medical and healthcare applications [3]. Generally, several countries suffer from dire shortages of healthcare personnel leading to a drop in the medical care quality and an extensive increase in healthcare costs. The competences of adapting new devices for health monitoring systems have risen. In healthcare monitoring, data transmission using low cost sensors and several communication media has directed to a foremost concern for the prevailing platforms leading to ineffectiveness in processing the massive data amount in real time. Consequently, in order to improve this field, an infrastructure and computing framework are required. This leads to Big data processing frameworks for Medical cyber-physical systems (MCPS) which integrate the cyber world aspects and the real world with dynamic, fully flexible systems for decision making and other healthcare applications [4] [5].

The current decade witnesses an innovative revolution in communication and computing. In widespread domains, the Internet has spanned numerous networks with significant impact on every life aspect especially in the medical domain. The social and economic impacts of the CPS and MCPS systems are immensely superior. Several investments are completed worldwide to improve this technology. Since the medical data may be transferred through wireless and/or social networks, the physical components of the devoted systems impose extensive challenges, such as reliability, security and safety requirements [6]. Such challenges are different from those in common computing applications. Furthermore, the physical components are qualitatively dissimilar to the common object-oriented software components. The next network generation will exploit a variety of resources with substantial sensing abilities to be extended beyond the physical connected computers in order to comprise multimodal information from cognitive, biological and social networks [7]. This standard modification will include interdependent social networks (networks of individuals), mobile personal computing, smart devices and communication devices, which will form cyber-physical social systems (CPSS) or net-centric societies (NCS). It becomes indispensable to determine the ability of the networking and computing technologies to provide a sufficient foundation for the MCPS [8] [9]. For alleviating the healthcare problems, technology development is considered a key role to improve the infrastructures using automated prevalent health monitoring technologies. Such technologies monitor person's health and alert the proper healthcare laborers in the emergency cases to offer the optimal care with nominal management.

Intelligent devices, including wearable devices and smart meters, with considerable sensing and networking capabilities become emerging. Henceforth, these devices, which are commonly known as the Internet-of-Things (IoT), will be persistently sensing, monitoring and interpreting the environment $[10,11]$. The association between the IoT and the social networks has an important implication for the innovative computing and for the communication infrastructure. Commonly, the IoT is limited to monitor and control small devices without any constraints to have the devices on a common network. However, the CPSs are more interested with how the physical systems are controlled and monitored using the cyber space. CPSs are exclusively identifiable and can be connected through the internet, which considered examples of the IoT. For information transfer, the IoT is basically networking of Cyber-Physical things. Therefore, CPS can be considered the first level and IoT and the second level of the vertical systems integration. Connected CPSs can be known as IoT. In order to provide real-time monitoring with feedback control services, sensing, processing and communication platforms can be deeply embedded in physical processes as a part of larger processes/systems. Such systems in the healthcare domain are called MCPS that has computing ability and a physical element to improve the healthcare via the comprehension pervasive health monitoring systems (PHMS). It supports the distributed computing using wireless devices. For smart healthcare, wireless body area networks (WBANs) have emerged as the foremost technology that offers ubiquitous healthcare services and real-time health monitoring $[12,13]$. The WBANs are considered medical cyber-physical systems that can operate in impenetrable environments, such as hospitals, leading to high interactive communication interference in several medical application scenarios. This requires provable behaviors or high-confidence and security.

The main contribution of the current survey is to provide a cutting-edge on MCPS systems and their role in the healthcare. The structure and platform of the CPS systems based on the WSNs are introduced as it is the milestone of ant MCPS systems. This survey highlighted the physical infrastructure required for CPS, the CPS general requirements and the related security issues. In addition, the CPS and MCPS in the social spaces are introduced. Furthermore, the networked medical device systems and IT-based social services in medical systems are also addressed. Finally, the recent research directions and challenges in MCPS for future medical devices are discussed. 


\section{Cyber-Physical Systems and medical CPS structure}

Enormous economic impairment occurs in the case of CPS malfunction which affects the corresponding systems operation. The CPS has physical infrastructure requirements along with networking models. The CPS becomes more complex, prevalent, personalized and reliable. The related systems can be used in several real-life applications, such as smart grids, robotics and healthcare. Improvements in the CPSs will empower the adaptability, capability, scalability, usability, resiliency, security and safety producing completely different system than the simplest embedded systems. The common CPSs characteristics include: i) have uncertainly regarding the status, readings, and trust; ii) must achieve real-time performance requirements; iii) have input and feedback from the physical environment without considering the communication channel; iv) include distributed management and control; v) have systems of systems control as well as multi-scale characteristics; vi) have geographical wide-distribution for components in locations that suffer from lack physical security.

In tele-medicine, for example, end users, i.e. doctors and patients, are connected through the internet. Thus, the high security internet connection is required during the patients' files/reports transfer. The control of un-/authorized flow of information with the consideration of physical and cyber consequences is required during the design of CPSs [14]. In addition, based on the application under concern, the communication architecture and the data must be designed to address all requirements involved. Fig. 1 illustrates the common data flow and interactions in CPS applications, which are also, can be applied to the MCPS.

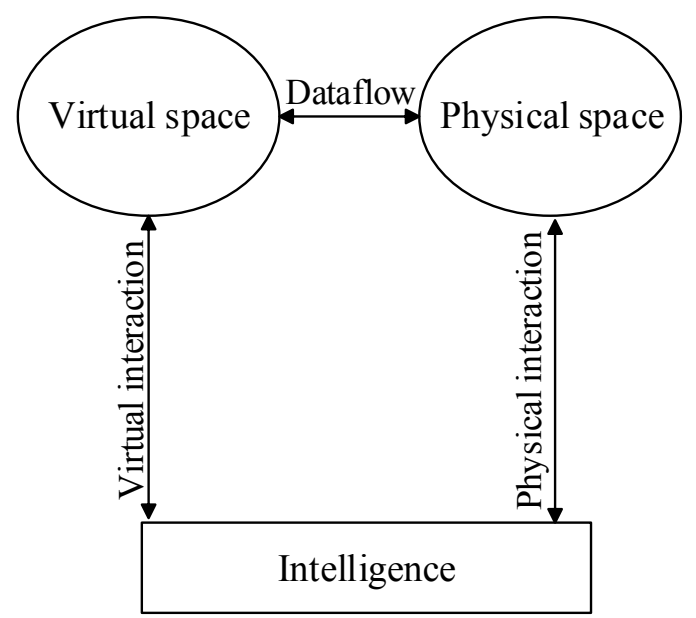

Fig. 1 Interactions in the CPS system

In addition, it is essential to understand the physical communication channels, i.e. the physical inputs from sensors, and the external-process channels like human operators' reactions with the system, wherein the MCPS, medical sensors and devices are included. For security and privacy in CPS and MCPS, high-confidence CPS and MCPS consider the emerging infrastructure challenge. However, the traditional analysis techniques are incapable to cope with both their complexity and predicting the system behavior. For instance, the MCPS requirements for security, dependability, privacy and safety grow immensely with the use of the enormous number of connected medical devices and sensors in the Internet of Things (IoT) systems during the interaction with the physical world and humans. Thus, proper technology is required to design and to hypothesize deep interdependencies for engineering systems. New relationships between the physical components and the cyber entail new architectural models, where the traditional real-time performance is inadequate for spatially and large CPSs. Since in the CPS and the MCPS as well, the feedback and input are from the physical environment, the presence of communication channels, which require security, is not usually considered. This is one of the CPS significant specific characteristic [15].

Incorrect specification of the information flow/accessibility requirements/control and/or implementation errors in the CPS and MCPS systems will lead to security failures. Security specification requirements should be considered for superior network development. Sensors security is an essential step in the CPS and MCPS systems. Procedures for tamper detection and input validation provided by the integrated sensors is significant to prevent recruited by adversaries in the control inputs to the cyber-physical system. Hence, the security is considered a part of the application development and system architecture. The assembly of data placement, monitoring and system control 
must consider the security implications [16]. Finally, the developed MCPS architectures and the system improvement tools are provided. Such isolation for security includes performance isolation, control isolation and data isolation within the virtualized distributed systems.

Embedded systems achieve higher predictability and reliability standard compared to the general-purpose computing. Thus, the integration of computing and the physical processes has a significant role, especially with the advancement of the embedded systems. Such incorporation leads to important applications, for example, in aircraft control systems, medical systems, weapons systems, communication systems and automotive electronics. Nevertheless, the embedded systems can be considered closed boxes that do not interpret the computing ability. The required fundamental transformation generated from networking the embedded devices faces several practical challenges. Several applications require networked and feature-rich embedded systems. The real-time dominant view has been well established before embedded computing. The current design of all operating systems, programming languages and computers may not fit the CPS and MCPS systems. The most extensively used networking procedures present the stochastic behavior and timing variability. Currently, the embedded systems use networking technologies. Cyber-physical systems are synchronized with nature and their integration with computing necessitates simultaneous composition of the computing methods with the physical ones. Embedded systems must respond to multiple real-time sensor stimuli streams and multiple actuator control simultaneously. Unfortunately, the interaction mechanisms with actuator hardware and sensors produces the interfere concept, which is not well signified in programming languages. Typically, a CPS requires improved predictability and reliability to be deployed in healthcare, automotive safety and traffic control applications [17]. Since the physical systems are not exclusively predictable, CPSs will not operate in controlled environments and must be flexible to subsystem failures and robust to unpredicted conditions.

In CPS and MCPS, a standard architecture can be considered in the social spaces. Cyber-physical systems progressively operate in social spaces, such as in the smart grid, disaster response systems and transportation systems where humans are the users, survivors or drivers. The main features of the CPSs include: functional integration, emergent intelligence, increasing complexity and adaptive behavior/structure leading to humans' huge impact on the environment. In a CPS, the users can be both in--the-loop and out-of-the-loop, where these systems have compatible characteristics $[18,19]$. The IoT adopts that things exchange information and interact to provide a foundation for future prevalent computing environments. Consequently, the CPS concept is delineated from the Internet of Things (IoT) as illustrated in Fig.2 [20].

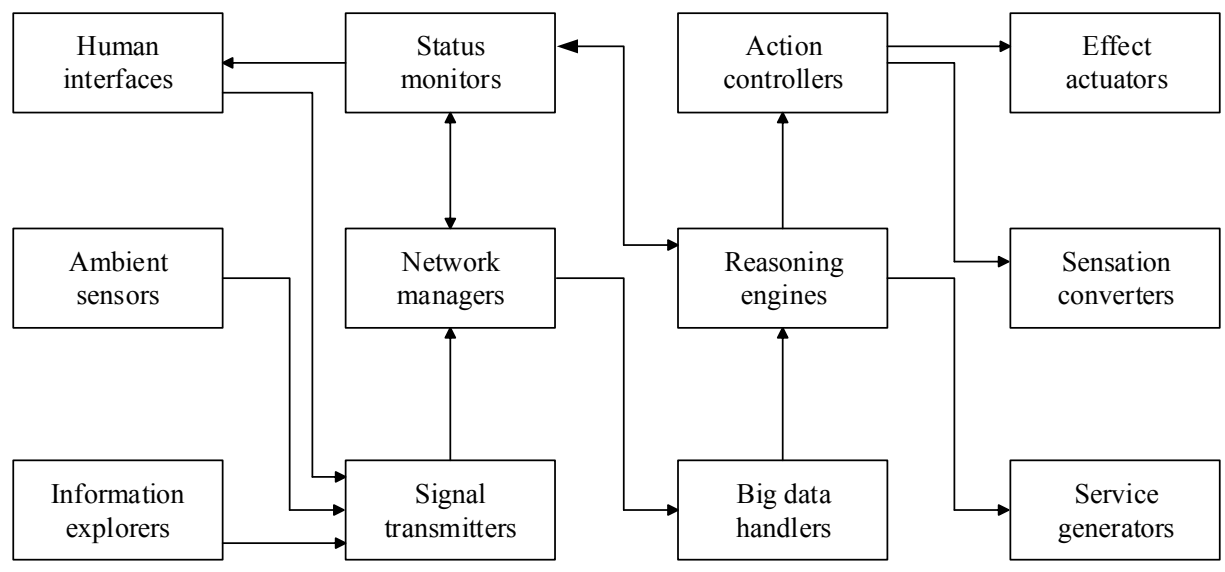

Fig.2. The cyber-physical systems standard architecture [20]

The CPS paradigm is still under progress; accordingly, different interpretations and implementations forms attract the focus of the researchers. Generally, CPSs and MCPS can be considered to be physical, engineered systems whose processes are monitored, controlled, coordinated and securely integrated by a communication and computing core at all levels and scales. It can be considered as two sub-systems, namely: i) cyber sub-system which is event-oriented, logic-based and discrete system that is responsible for communication, computation and control and ii) physical sub-system, which operates continuously and integrates the human-design and natural components that follow the physics laws. Subsequently, MCPSs have the dynamism, evolutionary and decentralization nature, where these systems are functionally and structurally open, intelligent, context-sensitive and self-adaptive engineered 
systems at which the physical and the cyber sub-systems evolve and interact cooperatively to increasingly penetrate into the social space as well as into the humans' world to support healthcare.

Generally, CPSs are gradually becoming part of the social-technical society fabric instead of being only just technical systems. They integrate the interaction between the embedded environments with the human domain forming two consistent socialization dimensions. These systems can be considered social-technical complex systems, wherein human and technical parts are immensely intertwined. Social-cyber-physical systems (SCPS) operate based on the humans' expectations, and the society/communities. These systems should have particular elementary social capabilities, namely: synchronizing their performance, accessing the data of users, sensing users and the social networks/associations between them, understanding the social goals based on the user model and social context, deducing the social context based on the networks topology of the users and providing a context driven output. The SCPS realization should encompass the social context which comprises a social person believes, culture, attitudes and the social interactions [21]. In this context, SCPS will have extra characteristics over CPS systems: i) the awareness ability of the users and their individuals and the social contexts, ii) can adapt themselves towards optimal collaboration, iii) the ability to accomplish the highest possible dependability level, security, accountability, maintainability, and accessibility and iv) the ability to attain a balance between the resources and the outputs expenses. However, data sources may be undependable in this context that is considered a challenge for CPS systems.

Advanced CPS applications are progressively operating in the social spaces at which the humans have a significant part in the inclusive system. Henceforth, CPS and MCPS imminent applications will be engineered with a consideration of the humans in the loop. The medical sensors can contribute to the patient's records, survivors can collect data on damage in the natural disaster cases and drivers can contribute data in the traffic blocking at various locations. Generally, the CPS in the social space can be employed in the various healthcare applications through the MCPS.

\section{Medical Cyber-Physical System}

Broadly, social media/space refers to Web-based facilities/tools that allow quick, easy and wide communication between individuals. It has great impact in the medical and healthcare applications as well as it provides a venue for interactive communication between the patients, physicians and health-organizations' staff quickly. It allows the transfer of the patients' files and records easily [22]. This leads to appraising role of the social media in healthcare applications. Consequently, SCPS becomes an undeniable force that has great influence on the medical sector; especially with the evolution in the medical device industry that employs the embedded software and the networking connectivity. With the advancement in the medical devices, it became interesting to replace the devices that designed to treat the patients independently of each other by integrating devices using distributed systems that concurrently control/investigate the patient's different physiological aspects. The combination of embedded medical devices, networking abilities and the complex physical dynamics transformed the medical system to contemporary medical device systems that considered a class of the cyber-physical systems, which call medical CPS (MCPS) [23]. Generally, there are several trends that emerged with the use of MCPS including [24] [25] [26]:

I. Improvement networking abilities: Currently, the medical devices' networking abilities are mainly used to monitor the patients via local connection of different medical devices to incorporate the patient monitoring and/or for remote monitoring as well as to interact with the electronic health records for storing.

II. New software-empowered embedded systems: Due to the new trends in the embedded systems, new functionality is motivated by the new software possibilities that developed the medical device systems.

III. The need for automatic monitor for the patient's status: Using an automated controller can deliver continuous observing of the patient's state and can handle routine situations.

IV. New inventions in the health care industry: The development in the healthcare devices, such as bio-sensors, accelerated the implementation of cloud computing and thus the social CPS systems.

V. Development of computer technology and wireless communication: Information technology, networking and broad mobile broadband networks, motivate the use of such portable devices to access the Internet services. Thus, mobile healthcare becomes more popular through the CPS systems. 
Consequently, the MCPS systems combine the embedded devices, controlling software and communication channel for interaction [27]. In the current medical technologies, the rapid progress led to a new healthcare generation as well as new treatment strategies. The new implemented devices include implantable/bio-compatible devices, electro-anatomical mapping, robotic prosthetics and invasive embedded devices. MPCS transform the monitoring and computation processes of the stand-alone system to remote monitoring system. There are several scenarios for the MCPS applications including analgesic infusion pumps, patient monitoring and implant sensor devices. The computerization of different medication/medical devices raises their reliability on a computer system for further real-time computation. These devices can be associated with computer network to eliminate the geographical barrier during the monitoring process. These medical devices comprise sensors that sense the different body parts to acquire the patient information for further access to computing devices for the manipulation and diagnostic decision-making. The new improvements in the medical sensors, WSN, and cloud computing provide the CPS a dominant candidate for several healthcare applications, such as in-home/in-hospital patient care [28]. These bio-sensors are capable of collecting vital patient information covering health data. Afterward, through the wireless communication medium, the collected data are transferred to a gateway. These gathered data from the sensors can be accessible to clinicians and stored in a server. Consequently, security becomes a vital issue, where the patient data are private. Therefore, for healthcare applications, special considerations are essential to guarantee data security while designing the CPS architecture.

Furthermore, healthcare applications necessitate computing resources for intelligent decision-making based on the patient data. The enduring research in the MCPS-based healthcare is endeavoring to use the cloud computing technologies as a backbone of the CPS systems to develop the system's scalability and to allow real-time data analysis. In the healthcare domain, the research on CPS is still in its early phases. In CPS, gathering the patient's input for effective decision-making. Such gathered input data includes i) active data, such as digital records and smart feedback system, and ii) passive input data, such as smart devices and/or biosensors in healthcare environments that can support the data acquisition process. This arrangement of data gaining and decision-making system yield to explores in the healthcare applications. Openings of exploiting CPS in healthcare comprise the use of coordinated interoperation of adaptive devices as well as new management concepts for the medical physical systems using control/computation along with body area networks, diminished implantable smart devices, new fabrication approaches and programmable materials.

The proposed CPS architectures for healthcare applications [29] are very limited, although many CPS architectures have been proposed. Wang et al. [30] offered a secured architecture for the CPS systems in healthcare. A WSN-cloud integrated framework was applied. Banerjee et al. [31] introduced the modeling and analysis of MCPS called CPS-MAS. Nevertheless, addressing the privacy and security issues was missed. The authors suggested CPS-MAS framework was interested with safety verification. In included various solutions for the addressed challenging problems of analyzing and modeling the complex nature of medical device interaction with the human body, transport delay, nonlinearity characteristics and the non-trivial interaction aggregation during the device networked operation. A drug delivery model example for chemotherapy and pain relief was addressed. Wu et al. [32] suggested an MCPS concept based on service-oriented architecture (SOA) without including the complete architectural context. Generally, the CPS applications in healthcare can be categorized into assisted- and controlledapplications. The former (assisted applications) comprise health monitoring without controlling the patient's normal living. It is used to offer medical advice by obtaining real-time physiological data through biosensors. Such assisted applications can be employed to support in-home elder people [33].

On the other hand, the controlled applications exist in intensive care and hospitals for medical support. The observation and monitoring level in the controlled environment is intense and high. Such applications exist in hospitals, where information from several sources, including biosensors, bedside monitors and physicians' observations are united to inform interventions. These networked closed-loop systems including humans in the loop can develop the medical workflows. In addition, emerging technologies enable the patients' remote care showing the effect of different activities of daily life for healthcare. Combining both the assisted and controlled aspects of healthcare transforms the healthcare system into a complex, large and safety-critical cyber-physical system with numerous advantages along with challenges [34].

Generally, healthcare progressively depends on medical systems and devices that are networked to match the patients' needs with special circumstances. The CPS research provides numerous openings and challenges in biomedical engineering, healthcare and medicine, including image-guided therapy/surgery, intelligent operating 
rooms/hospitals, biological assays fluid flow control and the physical/neural prostheses improvement. Devices such as oxygen and ventilator delivery systems for breathing support, infusion pumps for sedation and monitoring sensors for patient's conditions are used in several operating rooms. These devices when collected in a new system configuration, it matches precise procedural or patient needs. The challenge in such MCPS systems is to control methodologies and to improve systems for operating and designing these systems. Therefore, researchers are interested in developing networked medical device systems based on information technology (IT)-based social services in medical systems.

\section{Networked Medical Device Systems and IT-based Social Services in Medical Systems}

Electronic circuits' diminishment, microprocessors advancement, wired/wireless digital networking and new manufactured materials lead to the replacement of the analog electromechanical devices/systems by a diverse array of modern medical devices that based on information technologies for patient monitoring, diagnosis and treatment. Such medical devices are associated with other devices in complex configurations to structure systems of systems that extent scales from tiny to ultra-large geographically distributed electronic records systems using networking [35]. IT-enabled medical devices shift to control the passive devices by the human operator using computing systems. Thus, the actuators and embedded sensors are actively controlling the patient's physiological functions and processes. Modeling, communications, sensing, embedded computing and incorporation with physical elements lead to new cyber-physical systems. A CPS system achieves functionality levels, flexibility and efficiency.

Medical device systems are designed to improve the patients' health using the new facilities that modern health care systems provide including practical health services and medical sensors. Physicians and the medical staff use the embedded device control Smart Medical Devices (SMDs) to continuously interact with the patient or the human body. The MCPS systems are combinations of physical processes with computation which are monitored and controlled by the embedded systems. Generally, CPSs have positively affected a number of application fields including the healthcare, infrastructure, communication, manufacturing, transportation and robotics.

For control and distributed sensing, the medical device networking can be employed at many levels. Highly distributed medical-device systems guarantee high quality of service (QoS), while the ad hoc evaluation of network applications for medical devices has ensued. Ad hoc endeavors to incorporate provider processes and the exclusive medical devices lead to very insecurely coupled networks that convey data between devices though PDA, including via IR communication and Bluetooth, memory stick, barcoding and/or other social services technologies [36]. Obviously, there is an emerging necessity for realistically designed high-confidence medical device CPS architectures. Furthermore, the developments in the medical networked embedded-control systems are essential to guarantee a high level of QoS. Hence, privacy, security, robustness, extensibility, interoperability, mobility and patient safety should be evaluated.

\subsection{Evolution of Medical Devices}

The architecture of the medical devices includes wired and wireless interfaces that enable networked communication and medical reports transfer of patients' data. However, unintended results may occur during the data aggregation across devices which are designed to operate separately through the ad hoc network. In health systems, the distributed communication and advanced networking dominant role is to transfer the medical information efficiently through the network in different systems, including telemedicine, home healthcare services and online clinical analysis. The networked medical device systems require high-confidence design and manufacturing of complex medical device systems and software such as the implants, different modalities, nano/bio devices and invasive surgical devices [37, 38]. Through the design of the medical devices, the MCPSs have a significant role for realistically ensures the high-confidence medical device cyber-physical architectures. The development strategies are essential for the medical device manufacturer to generate advanced principled, end-to-end, engineering-based progress tools. Empower active cooperation is essential between industry, government and academia to build the supporting networking/standards and information technology frameworks for evolving MCPS systems. In the medical devices area, the embedded systems (ES) are significant as they have control functions and can communicate with the engineered systems as well as with patients. An ES can be considered a computer system with special-purpose that designed to accomplish devoted functions as part of a mechanical device. The device architecture in the MD systems' structure is highly exclusive and depends on professionals to offer input and assess 
outputs. Networks can stream data into medical records, which routinely mine for knowledge extraction that energies actions host of including computerized treatment.

\subsection{IT-based Social Services in Medical Systems}

Traditional social networks are Web-based; however, the CPS-based social networking comprises physical inputs to enhance the users' interactions. For examples, users can share their sensing information through mobile phones, including, for example, audio, location, motion and acceleration. Consequently, the user's mobility patterns, activities and locations become transferable information in the cyber world. Healthcare dramatic changes have driven social service incorporation to the lead of health policy and care delivery transformation. The information technology (IT) future relies on the developing terminologies, including the ambient intelligence and pervasive computing. The ambient intelligence refers to the communication technology prominence in smart buildings and future homes for healthcare. The SMDS is used to support health systems and medical methods to provide healthcare solutions [39]. Embedded systems and CPSs provided immeasurable advancement in healthcare. Embedded software is hidden in the hardware components or written in a coordinated way. The embedded systems involve the microprocessor inbuilt of tiny device to increase the automated devices intelligence in diverse applications.

\subsection{Emerging Medical Device Systems}

Emerging medical device architecture consists of wired/wireless interfaces to enable networked device communication to record patients' data. These devices require networked device systems or closed-loop, where patients can be connected simultaneously to different medical devices. Such devices can be too, for example, regulate the breathing, to deliver drugs, or to report a physiological status. These acquired heath information from the diverse medical devices and sensors have to be analyzed and aggregated in a coordinated way through IT-based social services. For example, individuals may subject to miscommunication, information overload, and fatigue. These aspects can be combined to contribute as an adverse patient outcome. In the operating room, many devices can provide life-support functions based on the provided information by different monitoring devices [40]. Recently, most of the medical devices include digital technology to collect and to aggregate the device information for further transfer if required to the healthcare providers for some action or to trigger an independent action by a device. The necessity to offer healthcare services in a home care situation, or telemedicine by remotely transport expert medical practice, or execute an online clinical lab analysis, further emphasizes the distributed and networking communication dominant role of medical information by transferring electronic health records through social services. Thus, involve control mechanisms and computing to the serious medical information communicated through networking forms an essential pre-requisite to high-confidence cyber-physical medical systems to support the implementation of medical systems. Multiple CPS devices must interoperate together in order to optimize the patient care smoothly at a high confidence level.

\subsection{Wireless sensor networks towards cyber physical systems}

A body area network (BAN) is also called body sensor network (BSN) or wireless body area network (WBAN), which is a wireless network of computing wearable devices. For health monitoring, wireless systems/wireless sensor networks comprise of several types of implantable, miniature or wearable sensors. These biosensors are able to measure the important physiological parameters, such as the blood pressure, respiration rate, heart rate, body and skin temperature, and the electrocardiogram. The acquired quantities are interconnected either through a wired or a wireless link to a central node, such as the microcontroller board or a Personal Digital Assistant (PDA), which then in sequence exhibit the giving information on the user's interface or transmit the accumulated vital signs to a medical center. Wireless sensor networks (WSN) and mobile ad hoc network (MANET) are active research areas [41]. Fig. 3 illustrates the common CPS architecture model [41]. 


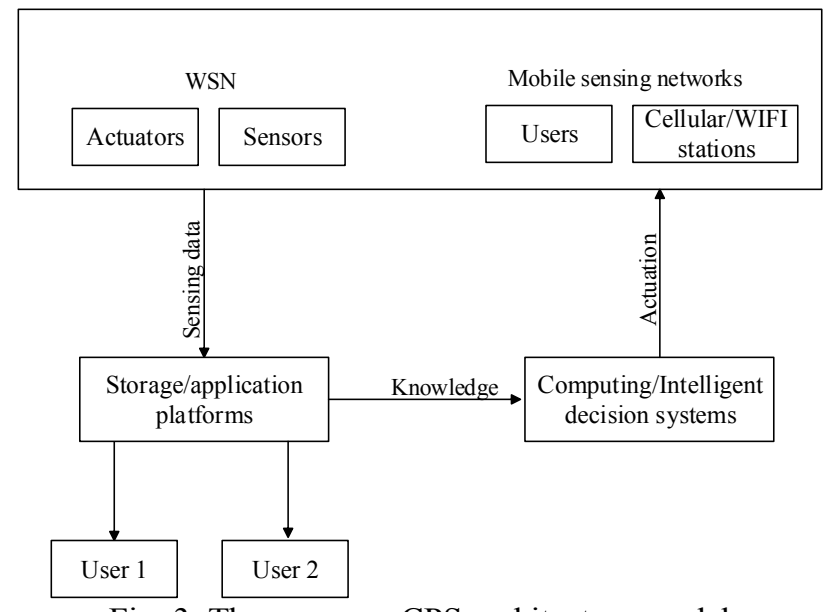

Fig. 3. The common CPS architecture model

However, the CPS systems are characterized by heterogeneous information flow, intelligent actuation/decision and cross-domain sensor cooperation. Thus, a CPS system can be employed effectively in several applications.

In addition, human-machine interaction is one of the interesting domains in the MCPS systems. The interaction between the humans and the medical devices/systems can be in two ways, namely humans as subjects or humans as operators, i.e., users [42]. Patient-specific modeling and simulation is required to empower patient-specific and adaptive therapies. Extensive approaches to design interfaces for enabling natural interaction by allowing: a) shared cyber and human understanding of the different system states (both failure and normal), b) accepting the actions, and c) sharing authority between the medical devices and humans.

\subsection{Machine-to-Machine Infrastructures toward Cyber-Physical Systems}

Substantial progress in many domains has arisen recently through wireless sensor networks (WSNs), wireless body area networks (WBAN) and machine-to-machine (M2M) communications. M2M denotes the communications between embedded processors, computers, mobile terminal devices, smart actuators and smart sensors with/without limited human interference [18]. The M2M communications have several advantages, including: i) multiple machines can efficiently interconnect in intelligent applications, and ii) the networked machine is more valued compared to the isolated one. Therefore, the M2M communications developer produces a several new opportunities for healthcare, information industry, smart home technologies, manufacturing systems and smart grids. Interfacing of M2M with WSNs, allow the M2M systems to assemble information via all sensors varieties. The M2M systems with decision-making capabilities and independent control can be promoted to an MCPS. In both the physical and virtual worlds, the CPSs have arisen as a promising route to enhance human-to object, object-to-object and human-to-human interactions. Actually, MCPSs would nurture and adopt WSNs and M2M, where further sensor inputs and richer network connectivity are required. Foremost differences between WSNs, M2M and CPSs are from the architecture and the design philosophy; even they are quite analogous in many networking features. Generally, WSNs are particularly designed to provide sensor-related data; M2M is used to support communications with/without limited human intervention, and MCPSs involve multiple sensing data dimensions, crosses the Internet/multiple sensor networks, highlights control functions and constructs intelligence across multiple domains. The MCPS systems are produced in M2M due to the development of advanced network techniques, distributed/real-time control, and cloud computing. They are characterized by having the cyber ability in every physical component and resource constraints, networked at extreme and multiple scales, are closely integrated, have complex multiple spatial and temporal scales, have continually reorganized/reconfigured, are high degrees of automation and closed-loop control, and have dependable and certified operation.

\section{Medical Cyber Physical Systems, Social Network and Big data Platforms}

Big data are high-volume, -velocity, and/or -variety information resources that entail new processing forms to empower improved insight discovery, process optimization and decision-making. It is referred to a structured and/or un- structured data collection that is produced by installed physical, mobile sensing devices, wireless sensor networks, remote (aerial) sensing, radiofrequency identification readers, chemical and biological sensors, software logs, software sensors, or any other data group facility of the CPS as well as the MCPS systems. This is merged with 
data that are encompassed in several socially assembled open sources that is freely accessible on the World Wide Web. Big data resources include images, video, text and sound. Big data can diver data from different sources, overwhelming amounts of data, data to be abstracted, and data can be used for semantic fusion. Typically, the big data design to support CPS requires new concepts to model the $4 \mathrm{~V}$ features, classical data structures, spatial constraints and the time constraints along with the dynamic continuous physical world performance.

The major related big data challenges are managing and exploitation. Information extraction requires qualitative and quantitative transformation of raw data in diverse contexts. Numerous CPS search engines apply computer learning mechanisms for this task. The produced big data type by the MCPS active components, the required target information as well as the learning procedures should be known. The synthesized or extracted information either remains interior in the system or becomes exterior information for the users. The extracted information needs to be communicated between the MCPS modules. Future big data management demands cost effective, advanced information processing forms for superior insight and decision-making [44].

The adaptation capabilities require a new class of devices for health monitoring system. However, the usage increase for sensors of low cost and several communication media in health monitoring have led to a chief concern for the prevailing platforms. The Medical Cyber Physical Systems (MCPS) are the system type that incorporates both the physical world aspects with the cyber space. The systems operate with a set of embedded devices and control these devices from cyberspace with a set of command and control statements. Any changes in the physical world are openly influenced the cyber space. In health care related applications, MCPS are commonly used. Small embedded systems can be attached to the human body in the remote healthcare monitoring system to capture various body conditions. Afterward, those collected information is processed locally in real times and only relevant information are sent back to the cyber space through internet or via mobile. The common wireless sensor architecture [45] for the Social PCS healthcare monitoring system is depicted in Fig. 4.

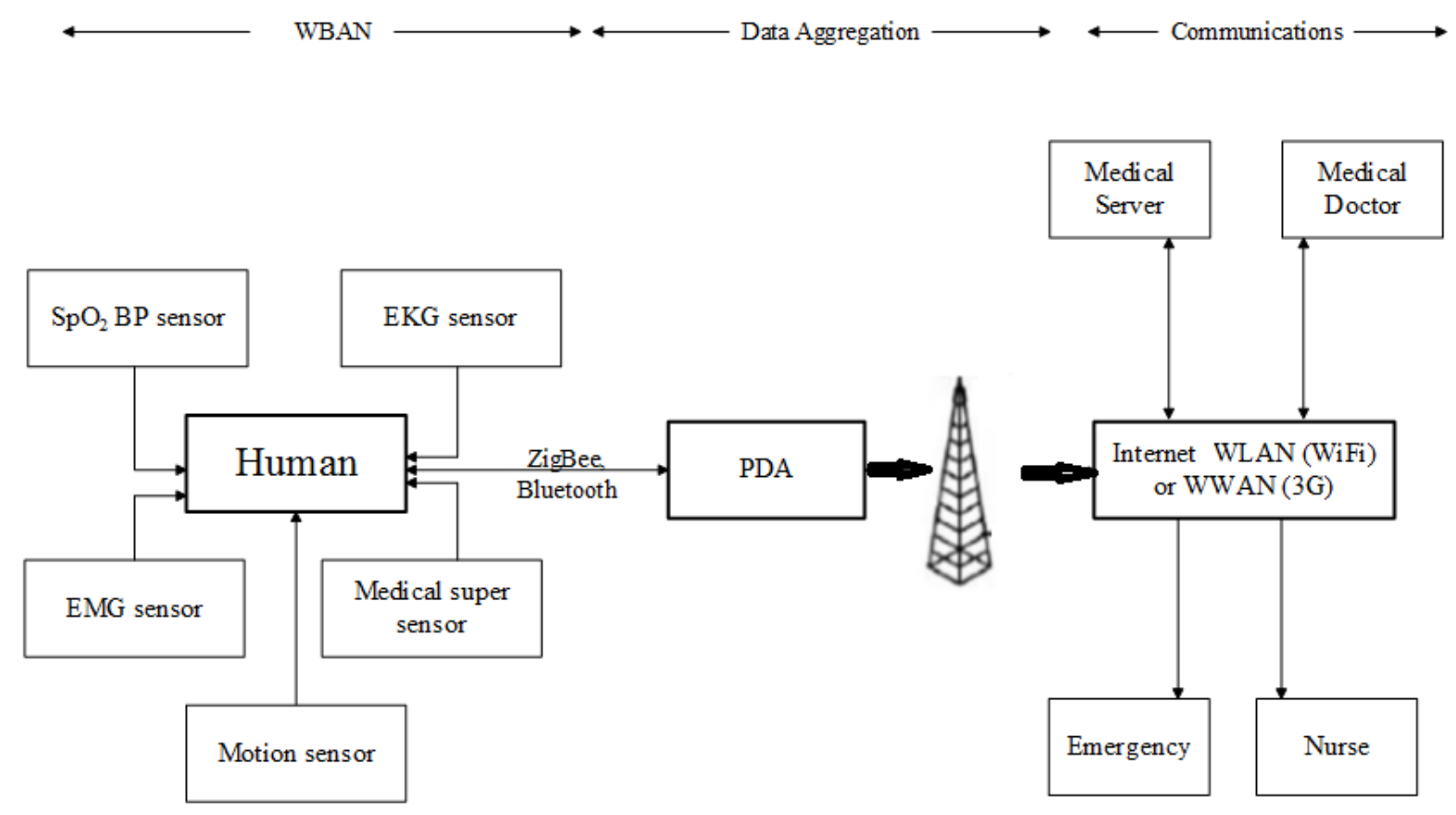

Fig. 4 Common architecture of wireless sensors for Social PCS healthcare monitoring systems

The CPSs have an imperative role in the social networking, big data analytics, and healthcare application analysis. The healthcare communities can face rising costs and severe medical personnel shortage. In order to alleviate this situation, the Pervasive Health Monitoring Systems (PHMS) can monitor the person's health continuously in real-time. The monitoring process is performed using a wireless network of ambient devices/sensors and medical, 
called Body Area Networks (BANs). The patient's privacy of collecting health information by PHMS is protected from acquiring the medical data from any unauthorized access. The CPS systems are computational platforms, networked, and extremely embedded in precise physical procedures to monitor and to actuate several purposes.

\section{Research Directions and Challenges in MCPS for Future Medical Devices}

Medical device systems are a leading example of cyber-physical systems, presenting complex and close sophisticated treatment algorithms interaction with the system physical aspects, and particularly the patient. The major challenge is to ensure and to develop the security, safety, and reliability of the CPS medical device, since such systems become increasingly interconnected, and complex [34, 44, 45]. Recently, social networks/services and big data analysis and applications magnetizes researchers [46-69]. Such interesting technology is employed recently to support healthcare applications through cyber-physical systems.

The CPSs can be considered a new pattern of IoT with the expansion of WSNs, M2M, radio frequency identification (RFID), inescapable computing technology, network communication equipment and emerging control model. The CPS applications have the impact to profit from enormous smart devices and wireless networks that tolerate the CPS applications to deliver intelligent facilities based on information from the surrounding physical world. Thus, one of the challenges is to provide high-performance CPS systems as a higher IoT phase. In addition, for the next generation of CPS systems, concurrent models of computation that are far more predictable, deterministic, and understandable will not be included. Several problems that inspire the researchers are focused on achieving advances in the formal verification, simulation and emulation techniques, certification techniques, software engineering processes, software component technologies, and the design patterns. To fully comprehend the CPS impact, the core computing abstractions need to be rethought with incremental improvements. However, effective software orchestration and the physical processes require semantic models to reflect the properties of interest. Some research challenges can be addressed as follows:

- Implementation technologies and platforms to support engineering- and science-based design and progress;

- New strategies for the progress of principled, end-to-end, and development tools.

For MCPS medical systems and devices, supplementary areas for consideration are:

- Synthesizing medical information, where the medical systems advance into CPS. This offers important challenges in capturing medical information as different physicians may predict varying treatment regimens for a given patient;

- In CPS-based healthcare systems, innovative services including schedulers to accommodate the reactive and synchronous system are required.

The emerging health cares supported by CPS have the impact to become the cost-effective laboratory/medical technology. Consequently, similarity in the devices' structure will be extremely dynamic based on the patient-specific medical concerns. This future will be depending on the technology foundation that can offer elastic configuration of embedded systems with control technologies and networking. Simultaneously, the information technology must manage optimized treatment delivery, diverse device requirements and stay highly approachable to the rapid changes in the users' requirements. For extremely integrated control and sensing, new opportunities are developing as the medical technology moves to smaller scales due to molecular and cellular dynamics prevail. In addition, more investigations should be directed toward the Holistic cyber-physical systems in the healthcare domain to build the next generations of medical CPS devices. On the other hand, CPS is considered an evolution of M2M by introducing more interactive operations and intelligent under the IoT architecture. However, the correlations among M2M, CPSs, WSNs, and the IoT is still an open research area.

The M2M is an active new field that has numerous significant challenges, namely: i) The cloud computing development will produce new perspectives to the M2M applications. Nevertheless, integrating the cloud computing with the M2M systems requires additional study; ii) Integrating M2M components with each other or with larger systems requires superior system integration skills; and iii) Creating M2M systems with compound mesh networks 
is expensive and complex requiring extensive study. Recently, other challenging directions concerning the CPS designs have arisen, including network security, energy management, data management/transmission, distributed real-time control, system resource management, model-based design and, platforms/systems. For forthcoming CPS systems, it is trial to maximize the QoS, while minimizing the energy consumption. Furthermore, the communication consistency is serious when manifold devices co-exist. For instance, Bluetooth, Zigbee, and WiFi operate usually on the same ISM frequency band to probably produce interference.

From the perspective of sociality, the present healthcare has several design concerns, including: (i) cyber and physical security, (ii) intelligent informing, (iii) access control, (iv) key management, encryption and secure protocols, (v) balance between availability and privacy, (vi) detection of physical and cyber-attacks data mining, and (vii) social contexts verification. In the context of MCPSs, there are different complexity types: (i) static complexity, where the components' relationships and number are unchangeable by time, (ii) dynamic complexity, where the components' relationships and number change with time, (iii) co-evolving complexity, (iv) evolving complexity and (v) self-organizing complexity. These complexities have to be resolved in future perspectives. The CPS is also powerful into human cognitive processes. For example, pattern recognition by humans and simplifying them into models is unclear and not implemented on computers. Finally, the recent promising domain of the healthcare applications includes: the medical Internet of Things (MIoT) to enable healthcare transformation, protecting interoperable clinical environment with authentication, and embedded, real-time, networked MCPS. Finally, several studies have been conducted on different Medical CPS applications that can be considered for further readings [7081].

\section{Conclusion}

The internet and computing revolutions opened up a huge set of new potentials in control that can impact human life through transportation means, new health treatments, new energy management and new services. These complex systems, which are networked, hybrid and integrated by different sub-systems, are cyber-physical systems that include human interactions as a central aspect. This consideration is applicable across the encompassing modeling, algorithm design, control space, operation, implementation and maintenance. Thus, human element needs to be rigorously analyzed, understood, and modeled. In this study, the framework of the cyber-physical systems in medical and healthcare domain was introduced. Cyber-physical systems excavate another thread, where the physical systems can be attacked through cyberspace, which in turn can be attacked through the physical devices. Thus, privacy and security are essential for both life quality and economic security. MCPS changes the dependable system design rules in the nature of faults. In the physical systems, it is assumed that the physical failures are liberated and that multiple, instantaneous failures are improbable. Cyber-attacks can be immense, presenting instantaneous or specifically coordinated failures. Generally, a considerable way for theoretical true MCPSs and SCPSs framework is essential. There is no combined design methodology to resolve the design challenges and management of the effects of long-term leaning and self-adaptation of MCPSs. There is a wide knowledge gap for the engineering and design technologies of comprehending high-end, non-linear CPSs that are interoperable, scalable and available. In order to advance the state-of-the-art, multidisciplinary operative knowledge synthesis is essential. The association between the M2M, WSN, MCPS, and the IoT is introduced. Finally, several challenges were addressed in almost all aspects related to the medical CPS.

\section{Compliance with Ethical Standards}

Funding: We are the authors confirm no funding obtained.

Conflict of Interest: We are the authors confirm that no conflict of interest.

Ethical approval: This article does not contain any studies with human participants or animals performed by any of the authors.

\section{References}

[1] Voit H (2013) An Arbitrated Networked Control Systems Approach to Cyber-Physical Systems (PhD Thesis), München, Technische Universität München, Diss.. 
[2] Yi MY, Fiedler KD, Park JS (2006) Understanding the role of individual innovativeness in the acceptance of it-based innovations: Comparative analyses of models and measures. Decision Sciences 37(3):393-426.

[3] Raghupathi W, Raghupathi V (2014) Big data analytics in healthcare: promise and potential. Health Information Science and Systems 2(1).

[4] Haque SA, Aziz SM, Rahman M. Review of cyber-physical system in healthcare. International Journal of Distributed Sensor Networks. 2014 Apr 27;10(4):217415.

[5] Hu L, Xie N, Kuang Z, Zhao K. Review of cyber-physical system architecture. InObject/Component/Service-Oriented Real-Time Distributed Computing Workshops (ISORCW), 2012 15th IEEE International Symposium on 2012 Apr 11 (pp. 25-30). IEEE.

[6] Li T. On coordination of cyber-physical systems (Doctoral dissertation, The Hong Kong Polytechnic University).

[7] Baronchelli A, Ferrer-i-Cancho R, Pastor-Satorras R, Chater N, Christiansen MH. Networks in cognitive science. Trends in cognitive sciences. $2013 \mathrm{Jul}$ 31;17(7):348-60.

[8] Liu Z, Yang DS, Wen D, Zhang WM, Mao W. Cyber-physical-social systems for command and control. IEEE Intelligent Systems. 2011 Jul;26(4):92-6.

[9] Rajkumar RR, Lee I, Sha L, Stankovic J. Cyber-physical systems: the next computing revolution. InProceedings of the 47th Design Automation Conference 2010 Jun 13 (pp. 731-736). ACM.

[10] Gubbi J, Buyya R, Marusic S, Palaniswami M. Internet of Things (IoT): A vision, architectural elements, and future directions. Future generation computer systems. 2013 Sep 30;29(7):1645-60.

[11] Alam S, Chowdhury MM, Noll J. Senaas: An event-driven sensor virtualization approach for internet of things cloud. InNetworked Embedded Systems for Enterprise Applications (NESEA), 2010 IEEE International Conference on 2010 Nov 25 (pp. 1-6). IEEE.

[12] Yilmaz T, Foster R, Hao Y. Detecting vital signs with wearable wireless sensors. Sensors. 2010 Dec 2;10(12):10837-62.

[13] Lee EA. Cyber physical systems: Design challenges. InObject oriented real-time distributed computing (isorc), 2008 11th ieee international symposium on 2008 May 5 (pp. 363-369). IEEE.

[14] Sampigethaya K, Poovendran R. Aviation cyber-physical systems: Foundations for future aircraft and air transport. Proceedings of the IEEE. 2013 Aug;101(8):1834-55.

[15] Neuman C. Challenges in security for cyber-physical systems. InDHS Workshop on Future Directions in Cyber-Physical Systems Security 2009 Jul 22 (pp. 22-24).

[16] Baheti R, Gill H. Cyber-physical systems. The impact of control technology. 2011 Mar;12:161-6.

[17] Kocsis I, Tóth ÁR, Szatmári Z, Dabóczi T, Pataricza A, Guta G. Towards cyber-physical system technologies over Apache VCL. International Journal of Cloud Computing. 2016;5(1-2):91-111.

[18] Wan J, Chen M, Xia F, Di L, Zhou K. From machine-to-machine communications towards cyber-physical systems. Computer Science and Information Systems. 2013;10(3):1105-28.

[19] Zhuge H. The Knowledge Grid: Toward Cyber-Physical Society. World Scientific; 2012.

[20] Horvath I. Beyond advanced mechatronics: new design challenges of Social-Cyber-Physical systems. InProceedings of the 1st Workshop on Mechatronic Design, Linz (Austria), 27-29 June, 2012 2012. ACCM Austrian Center of Competence in Mechatronics.

[21] Caplan MZ, Weissberg RP. Promoting social competence in early adolescence: Developmental considerations. Social competence in developmental perspective. 1989 Aug 31:371-85.

[22] Ackerman MJ, Filart R, Burgess LP, Lee I, Poropatich RK. Developing next-generation telehealth tools and technologies: patients, systems, and data perspectives. Telemedicine and e-Health. 2010 Feb 1;16(1):93-5.

[23] Arney D, Pajic M, Goldman JM, Lee I, Mangharam R, Sokolsky O. Toward patient safety in closed-loop medical device systems. InProceedings of the 1st ACM/IEEE International Conference on Cyber-Physical Systems 2010 Apr 13 (pp. 139-148). ACM.

[24] Qian L, Luo Z, Du Y, Guo L. Cloud computing: An overview. Cloud computing. 2009:626-31.

[25] Alexander K, Clarkson PJ. Good design practice for medical devices and equipment, Part II: design for validation. Journal of medical engineering \& technology. 2000 Jan 1;24(2):53-62.

[26] Goswami S, Roy P, Dey N, Chakraborty S. Wireless body area networks combined with mobile cloud computing in healthcare: a survey. Classification and Clustering in Biomedical Signal Processing. 2016 Apr $7: 388$.

[27] Lee I, Sokolsky O. Medical cyber physical systems. InDesign Automation Conference (DAC), 2010 47th ACM/IEEE 2010 Jun 13 (pp. 743-748). IEEE.

[28] Milenković A, Otto C, Jovanov E. Wireless sensor networks for personal health monitoring: Issues and an implementation. Computer communications. 2006 Aug 21;29(13):2521-33. 
[29] Hackmann G, Guo W, Yan G, Sun Z, Lu C, Dyke S. Cyber-physical codesign of distributed structural health monitoring with wireless sensor networks. IEEE Transactions on Parallel and Distributed Systems. 2014 Jan;25(1):63-72.

[30] Wang J, Abid H, Lee S, Shu L, Xia F. A secured health care application architecture for cyber-physical systems. arXiv preprint arXiv:1201.0213. 2011 Dec 31.

[31] Banerjee A, Gupta SK, Fainekos G, Varsamopoulos G. Towards modeling and analysis of cyber-physical medical systems. InProceedings of the 4th International Symposium on Applied Sciences in Biomedical and Communication Technologies 2011 Oct 26 (p. 154). ACM.

[32] Wu FJ, Kao YF, Tseng YC. From wireless sensor networks towards cyber physical systems. Pervasive and Mobile Computing. 2011 Aug 31;7(4):397-413.

[33] Taleb T, Bottazzi D, Guizani M, Nait-Charif H. Angelah: a framework for assisting elders at home. IEEE Journal on Selected Areas in Communications. 2009 May;27(4).

[34] Lee I, Sokolsky O, Chen S, Hatcliff J, Jee E, Kim B, King A, Mullen-Fortino M, Park S, Roederer A, Venkatasubramanian KK. Challenges and research directions in medical cyber-physical systems. Proceedings of the IEEE. 2012 Jan;100(1):75-90.

[35] Rajkumar RR, Lee I, Sha L, Stankovic J. Cyber-physical systems: the next computing revolution. InProceedings of the 47th Design Automation Conference 2010 Jun 13 (pp. 731-736). ACM.

[36] Kammer D, Lunsford EM, inventors; Palmsource, Inc., assignee. Power-conserving intuitive device discovery technique in a bluetooth environment. United States patent US 6,950,645. 2005 Sep 27.

[37] Fox RC. The evolution of medical uncertainty. The Milbank Memorial Fund Quarterly. Health and Society. 1980 Jan 1:1-49.

[38] Huebsch N, Mooney DJ. Inspiration and application in the evolution of biomaterials. Nature. 2009 Nov 26;462(7272):426-32.

[39] Idowu SA, Omotosho OJ, Ojesanmi OA, Maitanmi SO. Cyber-Physical Systems: A Framework for Prediction of Error in Smart Medical Devices. American Journal of Software Engineering and Applications. 2015;4(4):71-9.

[40] Pattichis CS, Kyriacou E, Voskarides S, Pattichis MS, Istepanian R, Schizas CN. Wireless telemedicine systems: an overview. IEEE Antennas and Propagation Magazine. 2002 Apr;44(2):143-53.

[41] Wu FJ, Kao YF, Tseng YC. From wireless sensor networks towards cyber physical systems. Pervasive and Mobile Computing. 2011 Aug 31;7(4):397-413.

[42] Levin E, Pieraccini R, Eckert W. A stochastic model of human-machine interaction for learning dialog strategies. IEEE Transactions on speech and audio processing. 2000 Jan;8(1):11-23.

[43] Jing Q, Vasilakos AV, Wan J, Lu J, Qiu D. Security of the internet of things: Perspectives and challenges. Wireless Networks. 2014 Nov 1;20(8):2481-501.

[44] Jiang Z, Pajic M, Mangharam R. Cyber-physical modeling of implantable cardiac medical devices. Proceedings of the IEEE. 2012 Jan;100(1):122-37.

[45] Otto C, Milenkovic A, Sanders C, Jovanov E. System architecture of a wireless body area sensor network for ubiquitous health monitoring. Journal of mobile multimedia. 2006 Jan 10;1(4):307-26.

[46] Tong GA, Li S, Wu W, Du DZ. Effector Detection in Social Networks. IEEE Transactions on Computational Social Systems. 2016 Dec;3(4):151-63.

[47] Nasim M, Charbey R, Prieur C, Brandes U. Investigating Link Inference in Partially Observable Networks: Friendship Ties and Interaction. IEEE Transactions on Computational Social Systems. 2016 Sep;3(3):113-9.

[48] Shmueli E, Singh VK, Lepri B, Pentland A. Sensing, understanding, and shaping social behavior. IEEE Transactions on Computational Social Systems. 2014 Mar;1(1):22-34.

[49] Farasat A, Gross G, Nagi R, Nikolaev AG. Social network analysis with data fusion. IEEE Transactions on Computational Social Systems. 2016 Jun;3(2):88-99.

[50] Guzman JD, Deckro RF, Robbins MJ, Morris JF, Ballester NA. An analytical comparison of social network measures. IEEE Transactions on Computational Social Systems. 2014 Mar;1(1):35-45.

[51] Yamin M, Sen AA. Improving Privacy and Security of User Data in Location Based Services. International Journal of Ambient Computing and Intelligence (IJACI). 2018 Jan 1;9(1):19-42.

[52] Belgharb K, Boufaida M. Using Event B to Specify Context Awareness for Service Discovery in Pervasive Environments. International Journal of Ambient Computing and Intelligence (IJACI). 2017 Jan 1;8(1):1-22.

[53] Sawlikar AP, Khan ZJ, Akojwar SG. Efficient energy saving cryptographic techniques with software solution in wireless network. International Journal of Synthetic Emotions (IJSE). 2016 Jul 1;7(2):78-96.

[54] Athavale N, Deshpande S, Chaudhary V, Chavan J, Barde SS. Framework for Threat Analysis and Attack Modelling of Network Security Protocols. International Journal of Synthetic Emotions (IJSE). 2017 Jul $1 ; 8(2): 62-75$. 
[55] Zhang W, Qi Q, Deng J. Building Intelligent Transportation Cloud Data Center Based on SOA. International Journal of Ambient Computing and Intelligence (IJACI). 2017 Apr 1;8(2):1-1.

[56] Jain A, Bhatnagar V. Concoction of Ambient Intelligence and Big Data for Better Patient Ministration Services. International Journal of Ambient Computing and Intelligence (IJACI). 2017 Oct 1;8(4):19-30.

[57] Chakraborty S, Chatterjee S, Dey N, Ashour AS, Hassanien AE. Comparative approach between singular value decomposition and randomized singular value decomposition-based watermarking. InIntelligent techniques in signal processing for multimedia security 2017 (pp. 133-149). Springer, Cham.

[58] Rajeswari P, Raju SV, Ashour AS, Dey N. Multi-fingerprint unimodel-based biometric authentication supporting cloud computing. InIntelligent techniques in signal processing for multimedia security 2017 (pp. 469-485). Springer, Cham.

[59] Koukopoulos D, Koukoulis K. A Trustworthy System with Mobile Services Facilitating the Everyday Life of a Museum. International Journal of Ambient Computing and Intelligence (IJACI). 2018 Jan 1;9(1):1-8.

[60] Roy P, Patra N, Mukherjee A, Ashour AS, Dey N, Biswas SP. Intelligent traffic monitoring system through auto and manual controlling using PC and Android Application. InApplied video processing in surveillance and monitoring systems 2017 (pp. 244-262). IGI Global.

[61] Kamal S, Dey N, Ashour AS, Ripon S, Balas VE, Kaysar MS. FbMapping: An automated system for monitoring Facebook data. Neural Network World. 2017;27(1):27.

[62] Wang D, Li Z, Cao L, Balas VE, Dey N, Ashour AS, McCauley P, Dimitra SP, Shi F. Image fusion incorporating parameter estimation optimized Gaussian mixture model and fuzzy weighted evaluation system: A case study in time-series plantar pressure data set. IEEE Sensors Journal. 2017 Mar 1;17(5):1407-20.

[63] Li Z, Dey N, Ashour AS, Cao L, Wang Y, Wang D, McCauley P, Balas VE, Shi K, Shi F. Convolutional Neural Network Based Clustering and Manifold Learning Method for Diabetic Plantar Pressure Imaging Dataset. Journal of Medical Imaging and Health Informatics. 2017 Jun 1;7(3):639-52.

[64] Wang C, Li Z, Dey N, Ashour A, Fong S, Sherratt RS, Wu L, Shi F. Histogram of oriented gradient based plantar pressure image feature extraction and classification employing fuzzy support vector machine. Journal of Medical Imaging and Health Informatics. 2017 Sep 8.

[65] Kamal MS, Parvin S, Ashour AS, Shi F, Dey N. De-Bruijn graph with MapReduce framework towards metagenomic data classification. International Journal of Information Technology. 2017 Mar 1;9(1):59-75.

[66] Kamal S, Dey N, Nimmy SF, Ripon SH, Ali NY, Ashour AS, Karaa WB, Nguyen GN, Shi F. Evolutionary framework for coding area selection from cancer data. Neural Computing and Applications. 2018 Feb 1;29(4):1015-37.

[67] Kamal MS, Chowdhury L, Khan MI, Ashour AS, Tavares JM, Dey N. Hidden Markov model and Chapman Kolmogrov for protein structures prediction from images. Computational biology and chemistry. 2017 Jun $1 ; 68: 231-44$.

[68] Dey N, Dey G, Chakraborty S, Chaudhuri SS. Feature analysis of blind watermarked electromyogram signal in wireless telemonitoring. InConcepts and trends in healthcare information systems 2014 (pp. 205-229). Springer, Cham.

[69] Dey N, Ashour AS, Chakraborty S, Banerjee S, Gospodinova E, Gospodinov M, Hassanien AE. Watermarking in Biomedical Signal Processing. InIntelligent Techniques in Signal Processing for Multimedia Security 2017 (pp. 345-369). Springer, Cham.

[70] Li W, Meng W, Su C, Kwok LF. Towards False Alarm Reduction using Fuzzy If-Then Rules for Medical Cyber Physical Systems. IEEE Access. 2018 Jan 17.

[71] Fu Z, Guo C, Ren S, Ou Y, Sha L. Modeling and Integrating Human Interaction Assumptions in Medical Cyber-Physical System Design. In2017 IEEE 30th International Symposium on Computer-Based Medical Systems (CBMS) 2017 Jun 1 (pp. 373-378). IEEE.

[72] Jiang Y, Song H, Wang R, Gu M, Sun J, Sha L. Data-centered runtime verification of wireless medical cyber-physical system. IEEE transactions on industrial informatics. 2017 Aug;13(4):1900-9.

[73] Gu L, Zeng D, Guo S, Barnawi A, Xiang Y. Cost efficient resource management in fog computing supported medical cyber-physical system. IEEE Transactions on Emerging Topics in Computing. 2017 Jan;5(1):108-19.

[74] Fu Z, Guo C, Ren S, Jiang Y, Sha L. Modeling and integrating physical environment assumptions in medical cyber-physical system design. InProceedings of the Conference on Design, Automation \& Test in Europe 2017 Mar 27 (pp. 1619-1622). European Design and Automation Association.

[75] Kocabas O, Soyata T, Aktas MK. Emerging security mechanisms for medical cyber physical systems. IEEE/ACM transactions on computational biology and bioinformatics. 2016 May 1;13(3):401-16.

[76] Mitchell R, Chen R. Behavior rule specification-based intrusion detection for safety critical medical cyber physical systems. IEEE Transactions on Dependable and Secure Computing. 2015 Jan 1;12(1):16-30. 
[77] Rao A, Carreón N, Lysecky R, Rozenblit J. Probabilistic Threat Detection for Risk Management in Cyber-physical Medical Systems. IEEE Software. 2018 Jan;35(1):38-43.

[78] Larson BR, Zhang Y, Barrett SC, Hatcliff J, Jones PL. Enabling safe interoperation by medical device virtual integration. IEEE Design \& Test. 2015 Oct;32(5):74-88.

[79] Ray A, Cleaveland R. Security Assurance Cases for Medical Cyber-Physical Systems. IEEE Design \& Test. 2015 Oct;32(5):56-65.

[80] Xu Q, Ren P, Song H, Du Q. Security-aware waveforms for enhancing wireless communications privacy in cyber-physical systems via multipath receptions. IEEE Internet of Things Journal. 2017 Dec;4(6):1924-33.

[81] Bernardeschi C, Domenici A, Masci P. A PVS-Simulink Integrated Environment for Model-Based Analysis of Cyber-Physical Systems. IEEE Transactions on Software Engineering. 2017 Apr 14. 